\title{
Margaret Mead
}

\author{
1941 \\ "À propos \\ des méthodes d'implantation \\ d'un programme \\ de conscience nationale"
}

Une traduction de Dimitri della Faille, septembre 2003.

Un document produit en version numérique par Dimitri della Faille, bénévole, Doctorant en sociologie à l’Université du Québec à Montréal

Courriel: della_faille_de_leverghem.dimitri@,courrier.uqam.ca

Dans le cadre de la collection: "Les classiques des sciences sociales" Une bibliothèque fondée et dirigée par Jean-Marie Tremblay, sociologue,

Site web: http://www.uqac.uquebec.ca/zone30/Classiques_des_sciences_sociales/index.html

Une collection développée en collaboration avec la Bibliothèque

Paul-Émile-Boulet de l'Université du Québec à Chicoutimi

Site web: http://bibliotheque.uqac.uquebec.ca/index.htm 
Cette édition électronique a été réalisée par Dimitri della Faille, bénévole, Doctorant en sociologie à l’Université du Québec à Montréal

Courriel: della faille_de_leverghem.dimitri@courrier.uqam.ca

\section{Margaret MEAD (1941)}

\section{“ À propos des méthodes d'implantation d'un programme de conscience nationale"}

Une édition électronique réalisée à partir de l'article de Margaret MEAD (1941), “À propos des méthodes d'implantation d'un programme de conscience nationale ". Traduction française de Dimitri della Faille, doctorant en sociologie à l'UQAM. Traduction de : Margaret Mead, " On Methods of Implementing a National Morale Program”, Applied Anthropology, Vol. 1, N. 1, October-December, 1941.

M. Dimitri della Faille nous autorise à diffuser la traduction de ce texte.

Courriel : della_faille_de_leverghem.dimitri@courrier.uqam.ca

Polices de caractères utilisée :

Pour le texte: Times, 12 points.

Pour les citations : Times 10 points.

Pour les notes de bas de page : Times, 10 points.

Édition électronique réalisée avec le traitement de textes Microsoft Word 2001 pour Macintosh.

Mise en page sur papier format

LETTRE (US letter), 8.5" x 11"')

Édition complétée le 16 septembre 2003 à Chicoutimi, Québec.

\section{\& Fait avec}




\section{Remarque du traducteur}

Margaret Mead (1941) « À propos des méthodes d'implémentation d'un programme de conscience nationale »

Traduction de: Mead, Margaret, "On Methods of Implementing a National Morale Program”. Applied Anthropology, Vol. 1, N. 1, October-December, 1941..

Margaret Mead est formée par la première génération d'anthropologues américains, en particulier par Franz Boas et Ruth Benedict. Elle occupe une place singulière au sein des sciences sociales américaines, c'est quelqu'un d'engagé et de virulent. Dans les années qui précédent la seconde guerre mondiale, elle lance avec son mari (G. Bateson) et R. Benedict l'école dite de la culture et de la personnalité aussi connue sous le nom de Culturalisme. En utilisant des explications anthropologiques, psychologiques et psychanalytiques, cette école tente de dégager le caractère propre de chacune des nations. De nombreuses sections de cet article en sont d'ailleurs des bonnes illustrations.

Mais ce qui caractérise davantage cet article, c'est son engagement en faveur d'une anthropologie appliquée. Cet article est d'ailleurs publié dans le premier numéro de la revue Applied Anthropology, revue officielle de l'association du même nom créée en 1941 par M. Mead ainsi que de nombreux autres anthropologues en réaction à des dissensions à l'intérieur de l'Association américaine d'anthropologie portant en particulier sur le type d'engagement décrit dans le texte ci-dessous que Mead adresse aux politiciens qui auront à statuer sur la manière de résoudre un conflit mondial, la seconde guerre mondiale, à partir des ressources locales. Le présent article est un plaidoyer pour la participation des anthropologues aux affaires publiques, et bien qu'il soit écrit dans le contexte de la seconde guerre mondiale, la Société pour l'anthropologie appliquée et ses membres tentent de s'assurer de la pérennité d'un certain pragmatisme et d'une professionnalisation de l'anthropologie aux États-Unis. 
Ceci est un article expérimental et ce de plusieurs manières. Celui qui se consacre à l'étude scientifique du social est confronté à la nécessité de tenter de traduire ses abstractions en règles pratiques et applicables. Il doit faire plusieurs efforts s'il entreprend une telle tentative. Il doit remanier ses abstractions sans ménagement parce que les outils afin de rendre applicables ses formulations raffinées n'ont pas encore été développés. Il peut prévenir une compagnie américaine travaillant dans l'exportation, que la couleur rouge a différentes significations dans la culture chinoise ou que les jumeaux ont un sens particulier pour les tribus ouest-africaines. Il peut alors suggérer de modifier l'usage de ces symboles dans la publicité que la compagnie désirait utiliser. S'il tente malgré tout de traduire son point de vue en actions dans la structure caractéristique ${ }^{1}$ - l'organisation caractéristique de l'expérience des différents peuples - il fera face à un problème que nous commençons à peine à démêler. Dans cet article, je fais l'hypothèse que nous ne pouvons pas présumer qu'il n'existe qu'une structure caractéristique unique proprement américaine. Les spécialistes objecteront immédiatement, mais dans cette société stratifiée, sectionnée, hétérogène et changeant rapidement, en divergence par rapport à ses sources européennes, toute déclaration quant à l'américanité 2 est vainement leste à moins que 1 'on prenne en considération ces facteurs ainsi que ceux de race et de religion. Mais, le problème que je me

1 Note du traducteur: Margaret Mead parle ici de Character Structure, qui se traduit difficilement en français. En effet, elle signifie la structure de ce qui est spécifique à un agent ou un à une institution. C'est son caractère, mais c'est bien plus, c'est également, la manière dont l'agent ou l'institution (peuples dans ce cas ci) réagit lorsqu'il est confronté à une situation ainsi que ce en quoi il se distingue de ses semblables. Aussi, au lieu de traduire Character Structure, par structure de caractère comme nous l'avons déjà lu, ce qui laisserait penser que la réaction est seulement le résultat du tempérament, nous avons choisi structure caractéristique, cependant, il aurait été préférable de l'appeler la structure idiosyncrasique pour insister sur la propension constructiviste de ce concept chez M. Mead ou dans l'école de la Culture et de la personnalité. Mais étant donné que cet article est envisagé par M. Mead comme un texte de vulgarisation, il est sans doute préférable de simplifier structure idiosyncrasique par structure caractéristique. Nous mettrons dorénavant structure caractéristique et caractère en italiques. Caractère américain signifiera dans notre traduction structure caractéristique aux Américains traduction de American Character. Toutes les notes de bas de page subséquentes sont du traducteur.

2 Pour respecter le texte, nous ne ferons pas de distinction entre l' « Amérique » et les « États-Unis », termes qui sont interchangeables et synonymes dans cet article de M.Mead. 
suis assigné est le suivant : comment l'anthropologue appliqué ${ }^{1}$ peut-il aider à implémenter un programme de conscience nationale ${ }^{2}$ ? J'ai pris pour acquis la structure politique actuelle de ce pays [les États-Unis], la tendance croissante à la planification centrale à partir de Washington ainsi que la possibilité que cette tendance s'accentue en cas d'urgence. Où, demandais-je, pouvons nous trouver la conscience nécessaire à une telle organisation? Ceci étant la première question, la seconde était : comment pouvons-nous inclure dans un tel plan un élément qui compenserait le réel manque d'intérêt pour les différences locales, de classe et de religion? Et finalement, il existe un problème auquel tous ceux qui envisagent la science appliquée font face, celui de la communication, rendre publique ses données. C'est un problème que j'ai déjà pu rencontrer par ailleurs. Aussi, ai-je tenté d'écrire la présente déclaration de la manière la plus intelligible qui soit pour les hommes d'État concernés par cette tâche.

L'organisation actuelle du programme de la Défense en comités locaux et d'État ${ }^{3}$, bien qu'elle mette l'emphase sur l'importance de la participation locale et volontaire, n'est pas conçue pour puiser de manière optimale dans les ressources du peuple américain. Ceci est dû à l'incapacité systématique de prendre en compte la structure caractéristique américaine ${ }^{4}$.

En 1941, pour établir une conscience locale nous devons agir avec les Américains tels qu'ils sont, et non pas tels que nous les pensons être. L'Américain typique réagit d'une manière précise à l'autorité venant d'en haut. L'Américain est extrêmement peu sensible aux leaders, aux appels à la Nation et aux demandes de faire partie d'un effort national si elles proviennent d'en haut. En Europe, tous les appels de ce genre sont basés sur une relation père-fils qui diffère de celle que nous avons en Amérique. L'enfant européen admire son père, il le craint et parce qu'il désire prendre sa place, il donne des ordres aux autres. L'enfant américain ne fait que tolérer son père; celui-ci est démodé, il ne parle que rarement anglais ou il ne connaît rien aux avions. Le petit Américain veut tout faire par lui-même, se trouver un emploi et se

1 M. Mead parle ici d'Applied Anthropologist, l'anthropologue qui envisage sa connaissance avant tout dans un sens pratique et applicable. Terme qu'il faut mettre en relation avec l'association (Society for Applied Anthropology) qu'elle vient alors de créer avec plusieurs collègues.

2 M. Mead parle ici de National Morale, le fait d'avoir confiance en sa nation et ses institutions et leur bien-fondé, d'avoir une conscience nationale, un esprit de corps, terme que nous traduirons par conscience nationale lorsque M.Mead parle de National Morale et par conscience lorsqu'elle utilise le terme Morale.

3 Le mot État réfère ici aux divisions politiques du territoire unies au sein du gouvernement fédéral, comme dans le nom «États-Unis».

4 Le soulignement est ici de M.Mead. Dans le texte, tous les soulignements subséquents sont aussi de M. Mead. 
débarrasser de la domination de son père qui dépend principalement du fait que ce dernier est le maître des cordons de la bourse. Toute son enfance durant, le petit Américain, c'est aussi vrai pour la petite Américaine, est intéressé, non pas à devenir comme ses parents, ce qui est la base de tous les nationalismes européens, mais est plutôt intéressé à se tenir avec ceux qui sont légèrement plus âgés et plus grands, il est intéressé à donner une raclée à ceux qui ont la même taille ou le même âge que lui et à prendre de l'avance sur ceux qui sont légèrement plus petits. L'étendue de sa compétitivité est très petite ; il n'est pas intéressé par les rêves de grandeur qu'ont les fils d'un père omnipotent. Être un « adulte ${ }^{1}$ signifie pour un américain de se débarrasser de toute supervision ; que papa ne puisse plus lui dire quand il a le droit d'emprunter son auto en possédant sa propre automobile... et que le gouvernement national n'y aie à y redire. Il ne veut pas se faire donner des ordres par en haut, ni par le gouvernement ni même par son propre père. Dépendre de son paternel signifie qu'il faille se faire dire à quelle heure rentrer; dépendre du gouvernement signifie, être assujetti à son aide d'une manière ou d'une autre.

Une analyse de la position des leaders en Amérique ainsi que des appels qu'ils doivent lancer démontrerait ce dernier point. Un candidat à la présidence se doit d'insister; comme tout le monde, il est né dans une ferme, il a cueilli du maïs et s'il a fait beaucoup d'argent, il est préférable que cela ne soit pas mentionné. S'il vient d'une famille occupant une position sociale supérieure, ce sera considéré comme un désavantage et il lui faudra une adresse particulière pour s'en débarrasser. Le peuple américain s'engouera plus facilement d'un candidat s'il pense celui-ci pareil à lui; à la différence qu'il est à peine plus chanceux d'en être arrivé là. Cela les rassure de savoir qu'il s'est rendu là; ils sont eux aussi faits de la trempe de ce que cela prend pour être Président. À défaut de tout cela, la campagne politique ne rejoindra pas leur imaginaire. Ceux qui sont nés à l'étranger, ceux qui appartiennent à des groupes touchés par la crise et ceux de minorités marginalisées, parce qu'atteints d'habitudes de dépendance et de désespoir, peuvent se rassembler autour d'un leader qu'ils identifient à un grand père blanc ${ }^{2}$. Ce n'est par contre aucunement le cas de l'Américain moyen. Cette tendance se confirme dans la manière dont ils élèvent les vedettes de cinéma au rang de symboles de succès. Ces vedettes ne sont pas particulièrement talentueuses ni belles d'ailleurs, elles sont des gens comme tout le monde qui ont percé. La pratique

Les guillemets sont ici de M. Mead. Dans le texte, tous les mises entre guillemets subséquentes sont aussi de M. Mead.

2 Traduction littérale de Great White Father, ici sans doute en référence au syndrome ou au mythe du même nom, celui d'un leader blanc autoritaire et paternel qui fait de son mieux pour « aider » les amérindiens qui, très naïvement, investissent en lui toute leur confiance. 
du leadership en Amérique implique que le leader s'identifie à l'individu américain moyen et non que le peuple s'identifie à lui.

Mais un programme de Défense avec un chef national et des comités d'État dirigés par des gouverneurs mis en place, devenus des représentants de Washington plutôt que du peuple de leurs États, essaie de tabler sur cet élément de faiblesse de la structure caractéristique américaine en disant aux adultes américains : «Ne désirerais-tu pas prétendre être un grand garçon et venir t'asseoir pour cinq minutes auprès de papa dans la première rangée ?» Parce que tous les Américains ont, un moment donné, été un enfant, si vulnérable et si petit, le plaisir de s'asseoir en avant avec papa est attrayant, mais il ne dure qu'une courte période. Tant dans les petites que les grandes villes, apparaît alors la réaction de ceux qui siégeaient aux comités de la Défense, ceux qui prétendaient aimer le faire. «Dieu du ciel, ne me parlez pas de Défense. » «Nous espérons réellement que l'orateur de ce soir ne mentionnera pas la Défense. » «Le sujet de la conférence suggère que nous n'aurons pas à entendre encore parler de Défense. » Cette lassitude et ce rejet sont le fait d'un enfant qui dirait : «Pourriez-vous m'excuser ? » et qui, aussitôt, s'éclipserait de la table où les adultes tiennent de lassantes et ennuyantes conversations. Le peuple américain est ennuyé par toute la mise en place de ce programme de Défense, tout autant que les Britanniques ont étés contrariés par les pannes de courant et les messages de précaution de l'ARP ${ }^{1}$ durant l'hiver 1939-1940. Tout cela provient d'en haut et n'a aucun fondement. Cela ne plait pas aux Américains car ils désirent faire les choses par eux-mêmes et qu'ils désirent FAIRE LA LEÇON ${ }^{2}$ aux gars du pâté de maison suivant, au prochain gardien, à la ville d'à côté, à l'autre troupe de scouts ou à l'équipe collégiale contre qui ils gagnent chaque année.

Un programme de Défense qui accomplirait quelques besognes à Oshkosh ou à Sioux City ${ }^{3}$ et qui fièrement, plein de condescendance, apparaîtrait dans un magazine ou un journal d'envergure nationale ne suffirait pas ${ }^{4}$. Le petit

1 ARP, Air Raid Precautions, L'ARP à été instauré en Grande-Bretagne dès 1935. L'on prévenait les civils de l'imminence d'une menace d'attaque aérienne grâce aux sons des sirènes.

2 La mise en majuscule est ici de M. Mead. Dans le texte, toutes les mises en majuscules subséquentes sont aussi de M. Mead.

3 Oshkosh se trouve dans le Wisconsin, la région des grands lacs du Nord des États-Unis et Sioux City, dans l'Iowa, la région du centre Ouest. Les deux villes ont une population amérindienne importante.

4 Ceci est sans doute une référence à la proposition de participation au Bureau des Affaires indiennes de la part des anthropologues Cole et Radcliffe Brown dès 1934. Ce bureau est (Office of Indian Affairs) dirigé par John Collier. Les anthropologues l'avaient contacté afin d'établir l'utilité de l'anthropologie pour le Bureau. En 1941, l'année de la publication de cet article, Collier annonce un plan de six années, en alliance avec des anthropologues, afin, entre autres, d'établir le profil de la personnalité des amérindiens. 
garçon qui lance la balle dans l'équipe locale de base-ball n'essaie pas de plaire à son père, ni même à ses oncles ou à ses grands-parents. Il désire que le reste de l'équipe dise de lui qu'il est bon, que les adversaires pensent que son équipe est chanceuse de l'avoir. Donner de la reconnaissance nationale à des groupes locaux, bien que cela soit temporairement gratifiant, n'est que de la condescendance de la part d'un adulte supérieur.

En d'autres mots, l'on ne donne pas de véritables racines aux plantes en repiquant des plants de tomates fanés à dix heures, le matin d'une chaude et sèche journée de juillet. L'image des racines d'une plante est une belle métaphore, mais elle ne veut certainement pas dire que l'on puisse transplanter une idée provenant d'une serre centralisante vers un conseil local mis en place par la Défense. La plante doit croître là, à même le sol local. Chaque ville, chaque établissement public, chaque église, chaque syndicat local doit sentir que «NOUS avons fait ceci et nous l'avons fait mieux que toute autre ville, tout autre établissement, toute autre église ou tout autre syndicat local. » Ils ne veulent pas être de factices émissaires du gouvernement national, ils ne veulent pas qu'on les prenne de haut. Ils veulent se sentir adultes, plein de vigueur et enclins à la tâche.

Qu'est-ce qui peut être fait pour utiliser pleinement toutes ces tendances du caractère américain?

(1) Il est nécessaire d'organiser des comités locaux autour de LEADERS NATURELS en lieu et place de ceux qui y sont à cause de leur position. Un gouverneur peut avoir été un leader naturel mais son placement peut aussi être le résultat d'une négociation politique. Dès qu'il occupe une position parce qu'il est gouverneur, c'en est trop pour la plupart des Américains. QUI est responsable de cette chose les gars ? Oh, les gouverneurs. Ohhh, (le son de la voix indique que finalement, ce n'est pas si important). Quelques hommes sont assez forts de sorte que lorsque les mots «LE MAIRE » sont dits, l'on songe à un individu en particulier, quelqu'un qui a un nom et une personnalité. Et ceci est bien fondé. Mais dans ce pays, vous devez être plus forts pour diriger en tant que maire ou que gouverneur que si vous désirez diriger sans pour autant occuper ces fonctions. À la question que se posent les Américains «Qu'a t'il fait pour être choisi ?» si la réponse est «ils choisissent, mais uniquement les maires et les gouverneurs » la réaction sera : «Oh, zut alors ! »

Pour faire en sorte que les groupes locaux prennent les tâches de Défense au sérieux, il faut que les leaders soient des leaders naturels. Ils peuvent être des maires ou des gouverneurs mais ils doivent être choisis pour eux-mêmes, 
par leurs propres communautés, et non pas parce qu'ils sont déjà à la tête d'une chambre de commerce ou d'un conseil de bien public. Les chicanes politiques et l'atmosphère assommante des bureaux entourant les fonctions officielles déteignent même sur les leaders naturels choisis par leurs propres groupes lorsqu'ils se trouvent placés dans un poste par une décision provenant d'en haut. Pour obtenir que les groupes locaux accordent leur confiance aux leaders nationaux, il est nécessaire d'avoir une équipe sur le terrain qui connaîtrait les manières de faire.

(2) Il est nécessaire de développer des règles de compétition et d'émulation locales, régionales et organisationnelles, celles-ci utiliseraient pleinement le désir qu'ont les Américains de montrer de quoi ils sont capables aux autres de même envergure. De telles règles requirent un peu de travail préliminaire, mais ce travail concerne de l'information qui, pour la plupart, existe déjà. Cela doit être organisé de manière simple. Les bureaux nationaux et régionaux devraient avoir en leur possession DES REPRÉSENTATIONS DES ORIENTATIONS DE LA RIVALITÉ NATURELLE concernant les villes de taille et certaines régions, comme les villes de la Nouvelle Angleterre ${ }^{1}$ ou de la Ceinture de maïs ${ }^{2}$ par exemple, ainsi que pour tout groupement volontaire important. Si la loge des Odd Fellows ${ }^{3}$ de Terre Haute ${ }^{4}$ veut faire quelque chose, l'équipe technique pourrait suggérer de parler de ses activités dans son bulletin et ainsi, de rejoindre d'autres loges de même envergure et de même force. Chaque nouvelle de tel type devrait être organisée de manière à jouer sur la rivalité existant à l'intérieur de son organisation. L'envergure et la force de la loge doivent être jugées adéquatement au préalable afin que chaque lecteur puisse effectuer les classifications de rigueur. "Elle est de notre catégorie, mais un peu plus grosse. Nous pourrions les battre. » ou «Bon dieu, une loge plus petite que la nôtre, nous ferions mieux de nous activer. »Ces deux commentaires sont les seuls que l'on peut envisager comme utiles. S'ils lisent des informations sur une loge bien plus grande, cela n'a aucun intérêt du point de vue d'une action.

Rien de tout ceci doit être laissé au hasard. Pour chaque bon plan, chaque invention, chaque fait marquant ou chaque activité identifiée au niveau local, il pourrait y avoir une règle définie de promotion qui tablerait sur ce type de rivalité. Dès que l'on joue sur la rivalité, trois niveaux peuvent être utilisés. Si

1 Région de la côte Nord Est des États-Unis, les États du Vermont, du Maine et du Massachusetts principalement.

2 Région du centre Ouest des États-Unis, les États de l'Illinois et de l'Iowa principalement.

3 Mouvement fondé à la fin du dix-huitième siècle dont le fonctionnement est proche de celui des Francs-maçons. Selon l'image habituelle, toute petite ville des États-Unis a sa chambre de commerce et/ou sa loge des Francs-maçons ou des Odd Fellows.

$4 \quad$ Ville de l'Indiana, la région du centre Ouest des États-Unis. 
une petite université de l'État de New York travaillant dans la proximité de sa communauté s'est particulièrement illustrée en assimilant 150 familles d'ouvriers qualifiés nouvellement arrivés, il faudrait que les collèges ${ }^{1} \mathrm{~d}$ 'autres villes de taille équivalente dans l'État de New York, peut-être même aussi de l'État voisin de Pennsylvanie, soient tenus au courant. C'est particulièrement important dans le cas de petits collèges qui participent à l'industrie de la Défense. Grâce à tout cela, deux types de rivalités vont être invoqués : « Nous pouvons battre les autres qui sont dans notre organisation» et «Notre organisation peut battre d'autres organisations. » L'église Baptiste locale pourrait entrer en compétition avec toutes les autres églises Baptistes de cet État, mais aussi avec l'église Méthodiste locale dont les activités motivées par le désir de dépasser l'église Baptiste locale seraient alors relatées auprès de toutes les autres églises Méthodistes de ce même État. Des représentations des RIVALITÉS ENTRECROISÉES seront utilisées lorsqu'il sera nécessaire de rendre publics les résultats.

\section{Que feraient ces comités locaux?}

Actuellement, l'on produit du découragement en grosse quantité parce que les communautés locales ne pensent qu'en termes de plans à l'échelle nationale: pour l'inscription nationale de volontaires, pour des comités de vigilance, pour collecter de l'aluminium. Ce sont tous des plans qui sont considérés par les institutions nationales comme prématurés ou peu raisonnables. Les institutions nationales doivent remercier les patriotes locaux tout en leur demandant d'arrêter pour l'instant. Il en résulte une impasse. Si l'action de la communauté locale doit renforcer l'action nationale, il y a plusieurs choses qui doivent être prises en compte.

(1) L'on doit pousser les communautés locales à travailler sur des problèmes réels devant être résolus; ces problèmes ont si possible plusieurs réponses. L'on peut par exemple assimiler des travailleurs de la Défense, préparer la grogne qui résulterait d'une carence de biens sur le marché, planifier l'achat groupé de bons de la Défense ou bien encore, travailler sur des plans adressés à la communauté qui donneraient des conseils d'alimentation. Toutes ces activités sont valables, il n'existe pas de réponse unique. De plus, lorsque l'on s'adresse aux groupes locaux, il serait préférable d'allier des tâches pouvant être achevées avec d'autres qui bien que non encore terminées

1 Un collège aux États-Unis est une institution locale d'enseignement supérieur qui délivre des diplômes semblables à la maîtrise française ou au baccalauréat québécois. Une université offre le même enseignement, mais elle est de plus grande taille; et contrairement aux collèges, elle propose la plupart du temps des chambres aux étudiants, des mastères (Masters) et des doctorats $(\mathrm{PhD})$. Cette différence tend actuellement à disparaître. 
puissent très bien être continuées dans l'urgence. Dans les suggestions que l'équipe de terrain ferait aux comités locaux, la proportion des différents types de problèmes devrait être adaptée à l'état de la conscience locale. Dans ce que le gouvernement cherche à expérimenter au préalable, si une conscience locale est très faible et hésitante, l'on pourrait suggérer deux ou trois tâches qui peuvent facilement être achevées et ce, de manière satisfaisante. Alors que la conscience se renforce, l'on peut envisager des tâches plus longues pour lesquelles un progrès est envisageable mais dont l'accomplissement de la tâche donne plus de sens que sa conclusion. Si, des hauteurs alors atteintes, la conscience devait dégringoler - ce qui risque d'arriver lorsqu'un état d'urgence en se stabilisant se dénuerait de toute excitation et ne provoquerait plus de montées d'adrénaline - l'on devrait introduire de nouveaux problèmes qui dépasseraient les seuls problèmes liés à cet état d'urgence. Les techniciens sur le terrain seraient entrainés à jauger le rythme de chaque communauté et à faire des estimations valables sur le type de projet qui conviendrait le plus à la conscience locale.

(2) Aucune communauté locale ne devrait travailler sur un problème qui aurait déjà été résolu par le gouvernement fédéral mais n'ayant encore fait l'objet d'aucune déclaration officielle. Par exemple, s'il est déjà décidé d'une altération ou d'une modification dans un plan, ni un forum, ni même une table ronde locale ne devraient pas être portés à se questionner sur ce problème. Ceci est simplement une manière de s'assurer que les plans sont effectués en toute bonne foi. Il serait de plus nécessaire de s'assurer que la machinerie administrative entend bien participer à ces plans dans ces conditions. Ces plans ne devraient pas être une manière élégante et détournée de faire exécuter par des communautés locales une évaluation de ce que le gouvernement fédéral aurait déjà décidé par ailleurs. Si une telle chose était entreprise, cela serait purement et simplement une autre forme plus vicieuse de manipulation de masse qui, tel un boomerang, se retournerait et aurait pour effet de diminuer la conscience, atténuer la confiance dans le gouvernement et démotiver l'initiative individuelle.

(3) L'on devrait demander aux groupes locaux de travailler sur des problèmes réels qui se déclinent en tendances déjà discernables. Par exemple, s'il apparaît que, dans une région donnée, la Défense doit former un camp de caravanes en réponse à une carence de logements, l'on doit tenir les groupes locaux des communautés où les industries de la Défense sont situées, mieux encore, où elles devraient être situées, qu'un camp de caravanes est la solution plutôt que de leur dire qu'ils doivent penser au problème de logement en général. À moins qu'une telle précaution soit prise, les groupes locaux vont dépenser leur énergie à inventer des solutions inutilisables résultant en une 
perte de temps et qui peut-être même affectera leur conscience. Mais si vingt groupes locaux se mettent à imaginer comment arranger les camps de caravanes pour qu'ils puissent s'établir tout en respectant les restrictions de la géographie locale, une part non négligeable de leur travail servira à l'établissement de la solution même si elle n'est pas nécessairement une addition essentielle aux plans prévus par le gouvernement. Lorsque le camp de caravanes sera installé, ils pourront au moins dire «nous avions prévu cela mis à part cette idée d'égouts empruntée par le gouvernement à ces hommes d'affaire de Lancaster. » Un sens de la fierté et de la réussite peut être ici combiné avec l'objectif de rivalité. «La prochaine fois qu'on nous demande de faire une tâche, nous allons montrer aux gens de Lancaster de quoi nous sommes capables. Cette fois-ci, la plupart de nos gars dormaient debout. Mais vous allez voir. »

(4) Si l'on envisage une utilisation complète de ces groupes locaux, ils devraient être encouragés à établir des rapports détaillés de leurs activités et à collecter des coupures provenant de la presse locale décrivant leur travail. Tout ceci fournirait une somme d'information sur la conscience qui pourrait alors être analysée par des experts qualifiés afin d'établir les tendances de la conscience dans le pays. De tels experts devraient bien sûr travailler à l'établissement de tests d'opinion adéquats qualitatifs et quantitatifs. C'est à dire des travaux de terrain sur l'état de la conscience ainsi que des sondages d'opinion plus généraux mais moins intensifs.

(5) Lorsque l'on rend publique toute solution à un problème trouvée par un groupe local, l'on peut alors insister sur ses meilleurs éléments, ceux-ci peuvent aussi être juxtaposés à l'explication d'autres aspects plus généraux pour lesquels cette solution est primordiale. L'on retirerait de l'exposé tous les éléments du plan qui paraissent assurément contradictoires ou impraticables. Si cela est fait dès la première étape, celle où un plan d'un groupe local est rendu public de manière à faire ressortir son appel potentiel à la rivalité naturelle, les groupes en compétition qui tentent de reproduire ce succès vont essayer d'améliorer cette solution, ainsi, ils travailleront à partir de quelque chose qui est déjà une amélioration des éléments d'origine. Cette technique fournirait une méthode rapide pour améliorer l'efficacité par l'intermédiaire d'un processus de mouvement progressif. Ce processus d'amélioration progressif serait en quelque sorte à l'exact opposé de la dégénération qui a lieu dans le jeu du téléphone arabe, lorsque les enfants s'amusent à se répéter des mots.

(6) Les limites de la rivalité resteraient fluides grâce aux REPRÉSENTATIONS DE RIVALITÉS ENTRECROISÉES dont nous avons discuté plus 
haut. Ainsi, le syndicat local ne sera pas seulement encouragé à continuer de participer à une chaîne sans fin d'émulation avec un autre syndicat local, avec une autre représentation du même syndicat ou avec une autre représentation du même syndicat dans une autre ville voisine et ainsi de suite. L'on devrait plutôt le pousser à mélanger les types d'émulation. Autrement, des situations sans issue risquent de mobiliser l'attention de groupes autour des sempiternels mêmes rivaux plutôt que d'utiliser leurs forces à la résolution de leurs propres problèmes.

(7) Les groupes locaux pourraient être utilisés à des fins de test, non seulement pour établir la description détaillée du fonctionnement d'une nouvelle politique, mais surtout, pour tester les difficultés liées à la conscience qui pourraient se développer autour de ces nouvelles politiques. À partir d'une connaissance du caractère américain et de la scène locale, l'équipe technique pourrait repérer et diagnostiquer les signes précurseurs d'un problème, suite à cela, elle pourrait s'adresser à l'agence fédérale concernée par ces plans pour que celle-ci puisse travailler sur la base de ces informations. L'on pourrait éliminer ce qui est le plus dommageable pour la conscience si on a le temps de pratiquer quelques essais préliminaires. Des plans impliquant un changement radical dans le régime alimentaire peuvent avoir des effets psychologiques secondaires imprévus qui risquent alors d'affecter la conscience. Des signes précurseurs de ces difficultés peuvent être décelés au cours de conversations à propos de projets locaux. L'on pense par exemple au cas de membres d'un comité local qui déclareraient ou révèleraient leur objection par des remarques du type «Ajouter des médicaments dans notre pain? Brrr. » Une fois qu'une politique est devenue un programme d'urgence nationale impliquant tout un investissement en planification et en prestige, il deviendra alors trop périlleux de pratiquer librement des expérimentations sans avoir d'opposition. Mais tant qu'une telle politique n'est discutée que par un comité local motivé par une volonté impétueuse d'aider l'établissement de politiques, toute expression de désapprobation, de dégoût, de répugnance, de mépris ou de peur serait bienvenue et ne risque pas de causer du dommage.

Quelles sont les grandes directions que ce programme prendrait? De tous les problèmes, ceci est, évidemment le plus grand. Il est impossible d'indiquer dans le détail ce que les groupes locaux doivent faire, ce qu'on doit leur dire, comment l'on formulerait leur effort jusqu'à ce que l'on ait une meilleure idée de la direction que les efforts nationaux doivent prendre. Mais si l'on travaille déjà sur la machinerie, qu'on l'apprête, elle pourrait utiliser sa pleine puissance dès que quelques renseignements pertinents seront révélés. Préparer la machinerie signifie former des équipes techniques de terrain et rassembler le matériel nécessaire pour établir les REPRÉSENTATIONS DE LA RIVALITÉ 


\section{NATURELLE et des plans de COMMUNICATION À PARTIR DE LA CONNAISSANCE DE LA RIVALITÉ NATURELLE.}

Travaillons maintenant quelques esquisses préparatoires à propos de la relation entre le caractère américain et les orientations nationales.

Si l'on décide que pour six autres mois, la Défense est encore une matière primordiale, l'on pourrait alors développer des manières significativement adéquates de traiter cette idée. Si nous nous préparons à rencontrer une menace, celle-ci ne remue pas encore suffisamment l'idée que l'Amérique se fait d'elle-même pour que cette préparation soit pressante. «Hitler est une menace, c'est-à-dire qu'il a un fusil plus gros que ceux que nous avons. D'accord! Mais, nous devons bâtir un plus gros fusil que le sien. Allons-y, mettons-nous à l'ouvrage. » Mais, ce n'est pas le cas. «Les gens ne se dépêchent que lorsqu'ils ont la frousse ou qu'ils doivent aller quelque part. » Les Américains n'admettront pas qu'ils sont effrayés et cela ne serait pas bon pour la conscience s'ils l'étaient. Et la Défense ne va nulle part, c'est comme si l'on payait une facture indue ou un impôt supplémentaire parce que deux années après avoir fait notre déclaration, le fisc découvrirait un oubli. Nous ne pouvons pas laisser la gestion de la conscience à un programme de Défense, mais c'est de surcroît risquer d'affecter la conscience que de le faire. Si nous utilisons des méthodes de pression pour intéresser à un contexte qui est essentiellement de la routine, qui n'est pas non plus attrayante, ni même plaisante et qui est de l'ordre de l'inéluctable, tout ce que nous faisons, c'est discréditer les méthodes. Dès qu'il peut être établi clairement que nous nous dirigeons vers un objectif, tel celui de gagner la guerre, et si un schéma de participation locale a été établi, l'on peut demander des Américains une pleine participation. Mais, même pour se rendre dans quelque direction que ce soit, pour redonner forme au monde ou pour faire disparaître Hitler de la carte peu importe le nom - la pleine participation des Américains ne peut pas être acquise par une pression mercantile ou une pression basée sur la propagande. En 1917, c'était une nouvelle expérience pour les Américains que de faire partie d'une entreprise d'envergure nationale. Parce que cela était une nouveauté, recevoir ses ordres directement de Washington et crier tous les mêmes slogans dictés par Washington. «Ceci sont les mots » était certainement attirant, mais, bien que cela fût invitant, cela ne dura pas longtemps parce c'était en inadéquation avec le caractère américain. Depuis lors, nous avons connu la désillusion de la dernière guerre et de sa propagande, des sempiternelles leçons concernant les publicitaires et la manière dont ils obtiennent leurs résultats, des efforts détaillés pour nous faire se méfier de la propagande allemande comme de toute propagande. Ceci n'est qu'un aspect 
de l'histoire. D'un autre point de vue, depuis la dépression ${ }^{1}$ des millions d'Américains ont été en contact avec Washington au travers de la WPA ${ }^{2}$ et des camps des $\mathrm{CCC}^{3}$ ! La relation entre Washington et une petite ville du Nebraska a cessé même d'avoir l'apparence attrayante qu'elle avait en $1917^{4}$. Cela ne marchera pas. Au lieu des « viens en avant Jimmy mon gars, viens avec Papa, prends le volant et fais semblant de le tenir pendant que je conduis », nous avons les « fais rouler doucement cette machine. Viens ici Jim et regarde donc si tu es capable de réduire la friction. » Nous devons penser à une manière d'agir rapidement. Nous avons du chemin à faire !

1 La grande dépression aux États-Unis qui commença avec la crise de 1929 et qui se termina avec la fin de la seconde guerre mondiale.

2 WPA, Works Progress Administration, ce programme lancé en 1935 par l'administration de F.D.Roosevelt dans le cadre de sa politique de la nouvelle donne (New Deal) visait à utiliser des personnes sans emploi afin de construire principalement des édifices, c'est le cas par exemple du pont du Golden Gate. Ce programme sera aussi entre autres utilisé pour le financement d'artistes comme en témoignent les gigantesques fresques de la Bibliothèque publique de New York.

3 CCC, Civilian Conservation Corps, créé par le Président F.D.Roosevelt en 1933 dans la même logique de la politique de la nouvelle donne. Ce programme enverra de très nombreux jeunes inoccupés effectuer des tâches rémunérées de conservation dans des régions rurales.

4 Probablement la ville d'Omaha, où M. Mead a effectué des recherches sur les amérindiens durant les années 1930. Si Mead fait référence au manque de participation de la population à la conscription de 1940, les États-Unis étaient alors encore en temps de paix, sa remarque n'a plus aucun sens dans les mois qui suivent la publication de cet article car, en effet, Omaha donne sa part de l'effort de guerre dès l'appel du Président. En plus de fournir son lot de soldats, de nombreuses cantines et camps de prisonniers y seront organisés. En outre, c'est l'usine Martin de cette ville du Nebraska qui, en plus de près de deux mille autres bombardiers, construira le bombardier Enola Gay qui servira à lâcher la bombe atomique qui détruisit Hiroshima. 